EPJ Web of Conferences 53, 04005 (2013)

DOI: $10.1051 /$ epjconf/20135304005

(C) Owned by the authors, published by EDP Sciences, 2013

\title{
HiRes and TA Composition measurements
}

\author{
Yuichiro Tameda ${ }^{\mathrm{a}}$ for the Telescope Array and HiRes Collaboration ${ }^{\mathrm{b}}$ \\ Institute for Cosmic Ray Research, the University of Tokyo, Kashiwa, Chiba, Japan
}

\begin{abstract}
In order to clarify the origin of ultra high energy cosmic rays (UHECRs), it is very important to determine the mass composition. The most effective strategy to determine the mass composition is $X_{\max }$ technique. $X_{\max }$ is the atmospheric depth of air shower maximum measured by fluorescence detectors (FDs). HiRes has reported $X_{\max }$ measurement by FDs which indicated proton dominated mass composition. Now, Telescope Array (TA) experiment has also measured UHECRs with FDs. In this presentation, the detail of mass composition analysis and result of TA experiment will be reported and compared with HiRes experiment.
\end{abstract}

\section{INTRODUCTION}

\subsection{High Resolution Fly's Eye}

The High Resolution Fly's Eye Experiment (HiRes), located on Dugway Proving Grounds in western Utah, is a UHECR observatory operated from 1997-2006. The experiment consisted of 2 sites, HiResI and HiRes-II, separated with $12.6 \mathrm{~km}$. It can measure extensive air showers (EASs) of UHECRs stereoscopically by the atmospheric fluorescence technique. The fluorescence telescope in each site has spherical mirror with area of $5.2 \mathrm{~m}^{2}$ to collect fluorescence light. At the focus of it, a cluster of photomultiplier tubes (PMTs) arranged in $16 \times 16$ array is mounted. Each PMT covers one degree sky and field of view (FOV) of the telescope is $14^{\circ} \times 16^{\circ}$. The telescopes are arranged as a ring to look around: HiRes-I has 21 mirrors in one ring with elevation angle of $3-17^{\circ}$ and HiRes-II has 42 mirrors in two rings with elevation angle of $3-31^{\circ}$. For the data acquisition (DAQ) system, HiRes-I uses sample and hold electronics, on the other hand, HiRes-II adopts FADC system.

\subsection{Telescope array}

The Telescope Array (TA) is a hybrid detector consisting of a Surface Detector (SD) array and Fluorescence Detectors (FDs) to observe UHECRs. It is located in the western desert of Utah, $\sim 100 \mathrm{~km}$ away to the south from HiRes site. The SD array consists of 507 three square meter plastic scintillation counters arranged on a $1.2 \mathrm{~km}$ grid for a detection area of $\sim 700 \mathrm{~km}^{2}, 7$ times larger than AGASA. There are three FD stations overlooking the SD array. Two of the FD stations, located at Black Rock (BR) and Long Ridge (LR), contain 12 newly developed FD telescopes each, the other, Middle Drum, contains 14 telescopes transferred from HiRes-1. TA has been taking data with all detectors since May 2008. The optics of FD at BR and LR consists of 18 spherical mirrors with $6 \mathrm{~m}$ curvature radius, $3 \mathrm{~m}$ diameter and the mirror area is $6.8 \mathrm{~m}^{2}$. At the mirror focus, there is a PMT cluster which contains 256 PMTs with BG3 (Schott) filter and is covered with acrylic window (paraglas). The FOV of each telescope is $18.0^{\circ}$ in

\footnotetext{
ae-mail: tame@icrr.utokyo.ac.jp

${ }^{b}$ For the full authorlist see Appendix "Collaborations" in this volume.
}

This is an Open Access article distributed under the terms of the Creative Commons Attribution License 2.0, which permits unrestricted use, distribution, and reproduction in any medium, provided the original work is properly cited. 
azimuth and $15.6^{\circ}$ in elevation, each PMT looks $1.1 \times 1.0^{\circ}$. The total FOV of each station is $108 \times 30^{\circ}$. Air showers of which primary energy is $10^{19} \mathrm{eV}$ and shower core is $30 \mathrm{~km}$ apart from the FD telescope can be triggered, thus such air showers whose cores are within the SD array can be observed by 2 FD stations, stereoscopically.

\subsection{Event reconstruction}

For the mass composition analysis, TA FD and HiRes use stereo air shower events measured by 2 FD sites. The accuracy of stereo shower geometry is much better than that of monocular. In the case of TA FD, the determination of arrival direction is $1.9 \mathrm{deg}$ for stereo mode, or $6.2 \mathrm{deg}$ for monocular mode. The procedure of event reconstruction for stereo events consists of mainly tow parts, geometrical reconstruction and shower profile reconstruction as following, especially for TA FD case. For the geometrical reconstruction, at first, shower detector plane (SDP) of each FD station is determined, which includes shower axis and the detector position which is defined as the center of FD station. Next, shower axis is determined as the intersection of the SDPs determined as above.

Once the geometry of the shower axis is determined, profile of shower development is reconstructed by Inverse Monte Carlo (IMC) technique [1] using the intensity of injection photons at the detector. IMC takes contribution of direct or scattered Cherenkov light into account. Shower profile is assumed to be fit with Gaisser-Hillas function and energy is estimated as an integration of it. For proton with energy above $10^{19} \mathrm{eV}$, the energy determination is $1.6 \pm 7.6 \%$ and the $X_{\max }$ accuracy is $-6.2 \pm 22 \mathrm{~g} / \mathrm{cm}^{2}$. The atmospheric profile used is the monthly average of the radiosonde launched at Elko, Nevada, which is the closest launch site to TA. The distribution of aerosols was measured at the TA site by LIDAR [2]. The total energy deposited which is calculated by integration of the Gaisser-Hillas function along the shower axis is $93 \%$ for protons and $89 \%$ for iron.

\section{MASS COMPOSITION ANALYSIS}

\section{1 $X_{\max }$ technique}

The longitudinal development of a UHECR EAS depends strongly on its primary energy and particle type. The depth in the atmosphere at which the number of particles in the shower reaches a maximum, $X_{\max }$, is a good indicator of primary particle type. Since FDs observe longitudinal development of air showers, this technique has the advantage over SDs of measuring the energy calorimetrically and determining primary particle type. However, primary particle type of EAS cannot be determined shower by shower due to fluctuations in development of individual showers. Thus, the mass composition should be determined on a statistical basis by comparing the $X_{\max }$ distribution of the data and expected from a Monte Carlo (MC) simulation. Here, it should be noted that the uncertainty of the MC depends strongly on hadron interaction models that have been extrapolated from measured cross sections at much lower energies.

An expected distribution of $X_{\max }$ is estimated with a MC shower simulation using CORSIKA [3]. QGSJET-01[4], QGSJET-II[5] and SIBYLL[6] are used for the hadronic interaction models. Primary particles are assumed to be either protons or iron nuclei. The left side of Fig. 1 shows the averaged $X_{\max }$ of each energy based on only the shower MC simulation. As energy increases, the $X_{\max }$ of air showers increase. At a given energy, the $X_{\max }$ of a light primary particle will be deeper than that of a heavy primary particle. Since the FDs only can see showers in certain geometric regions, $X_{\max }$ may be either above the FOV or below it, or it may be inside the field of view but the FD cannot reconstruct the shower (for instance, the shower may be coming nearly directly toward the FD). Moreover, reconstruction bias may affect $X_{\max }$ distribution, systematically. This means that the distribution of observed $X_{\max }$ can be different from the expected distribution estimated only by shower simulation such as CORSIKA. This means that actual FD configuration and reconstruction method should be take into account. 

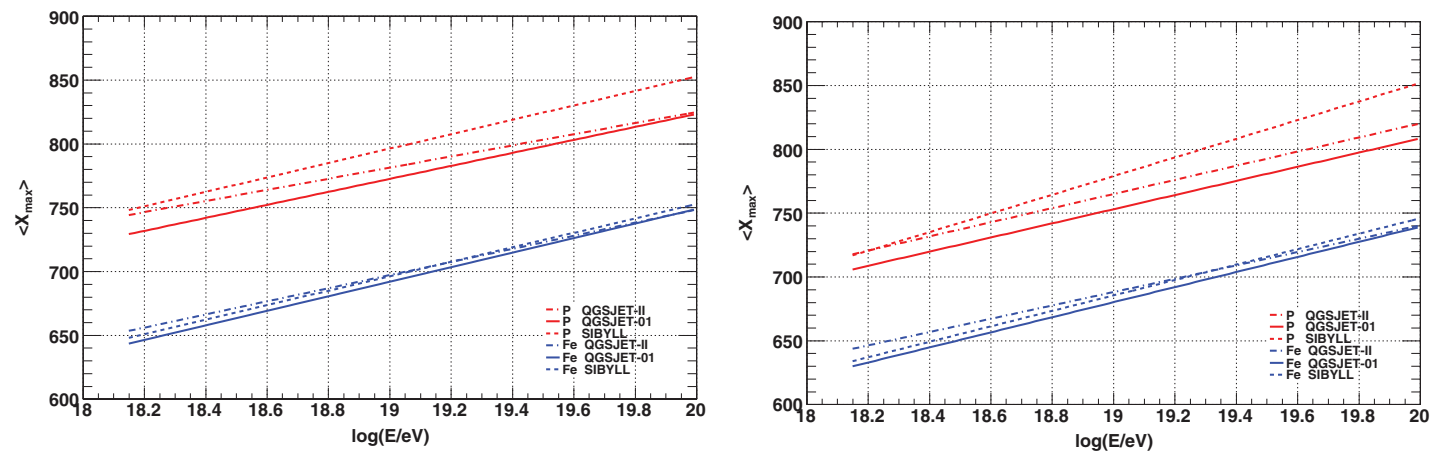

Figure 1. Averaged $X_{\max }$ of various hadronic interaction models: QGSJET-01, QGSJET-II and SIBYLL. In left figure, averaged $X_{\max }$ rails are derived from only shower Monte Carlo simulation. In right figure, TA FD detector response is taken into account.

The right side of Fig. 1 shows the averaged $X_{\max }$ in which the actual TA FD configuration are taken into account using detector simulation and the same reconstruction procedure as data is applied. Averaged $X_{\max }$ rails are shifted down systematically $20 \mathrm{~g} / \mathrm{cm}^{2}$ due to detector effect and reconstruction bias in TA FD case. In the case of HiRes, we can see the similar systematic shift of $16 \mathrm{~g} / \mathrm{cm}^{2}$ and it comes from detector effect called as acceptance bias. Reconstruction bias can be negligible in HiRes case. As above, $X_{\max }$ technique depend on the detector simulation, which should be understood well. Agreement between data and $\mathrm{MC}$ can be one of good indicator to evaluate our simulation performance.

Figure 2 shows the comparison of data and MC above the energy of $10^{18} \mathrm{eV}$ in HiRes case for distribution of several parameters of zenith angle, height of $X_{\max }$, first viewed depth and last viewed depth for two primary particle type of proton and iron nuclei. Hadronic interaction model shown here is QGSJET-II. We can see good agreement between data and MC, especially for proton primary model. In case of iron, differences can be found. The comparison of zenith angle distribution of data and MC with TA SD shows similar tendency with much higher statistics. Figure 3 shows the TA FD stereo case for several parameters of zenith angle, azimuth angle, core location, impact parameter $(R p)$ and track length. As above, we check carefully the detector simulation by comparison of data and MC to evaluate our analysis.

\section{$2.2 X_{\max }$ distribution}

Figure 4 shows the $X_{\max }$ distributions compared with MC based on QGSJET-II above energy of $10^{18} \mathrm{eV}$. Left of Fig. 4 are HiRes case compared with proton (upper) and iron (lower) model. Right of Fig. 4 is TA FD stereo, proton (red) and iron (blue). We can see good agreement between data and MC with proton primary model for each experiment. We applied Kolmogorov-Smirnov (KS) test to estimate the degree of agreement of the $X_{\max }$ distribution in each energy region. Figure 4 are the results of KS test. Each of them, in the whole energy regions, the $X_{\max }$ can be compatible with proton primary model. At the low energy, iron model can be excluded but at high energy, iron model can be also compatible with data due to low statistics.

Comparison of averaged $X_{\max }$ plot is obvious way to determine the mass composition of UHECRs. Figure 6 show the results of averaged $X_{\max }$ of HiRes (left) and TA FD stereo (right). In each figure, $X_{\max }$ rails are derived from shower simulation with detector simulation; the same as the left of Fig. 1 for TA FD case. The results of both experiments show that data is consistent with proton primary model, especially QGSJET model in whole energy region. 

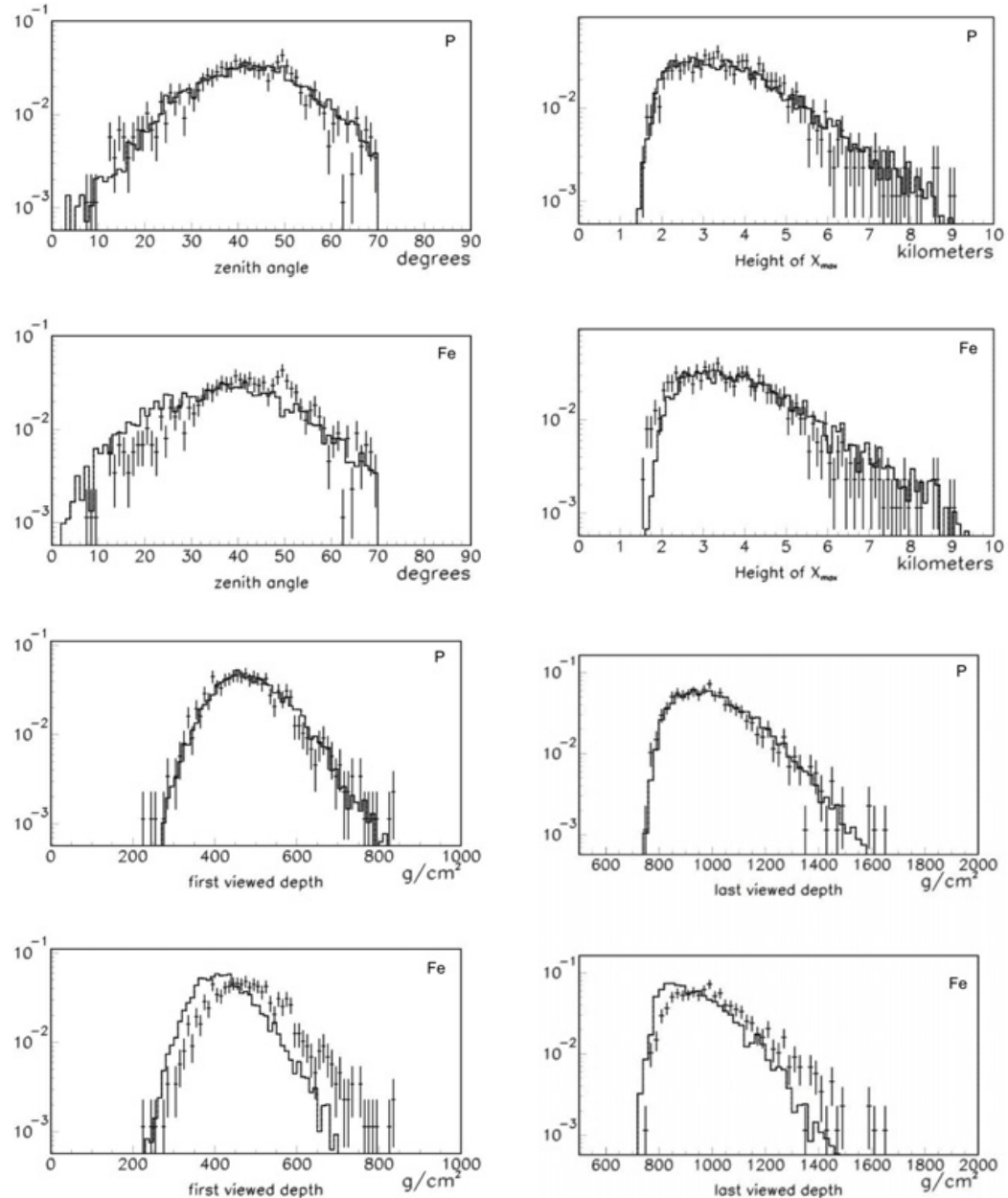

Figure 2. Comparison of the data and MC for HiRes events: zenith angle (upper left), height of $X_{\max }$ (upper right), first viewed depth (lower left) and last viewed depth (lower right). For each parameter, upper and lower figures are proton and iron primary case, respectively.

\section{SUMMARY}

In this presentation, the HiRes and TA results of UHECR mass composition is presented. Mass composition analysis derived from $X_{\max }$ technique is affected by acceptance or reconstruction bias which is well understood by comparison of data and MC. $X_{\max }$ distribution is consistent with proton primary model with QGSJET model above $10^{18} \mathrm{eV}$. $X_{\max }$ distributions for each energy are tested by KS test and $\mathrm{P}$ values show that proton model is compatible with proton model for whole energy region. On the other hand, iron model can be excluded, but below $10^{19.4} \mathrm{eV}$ for TA case. Averaged $X_{\max }$ shows that data is consistent with proton model, especially QGSJET model. Both TA and HiRes results of the UHECR mass composition are consistent with proton model from not only the distribution of $X_{\max }$ but also averaged $X_{\max }$ above $\sim 10^{18.2} \mathrm{eV}$. 
UHECR 2012
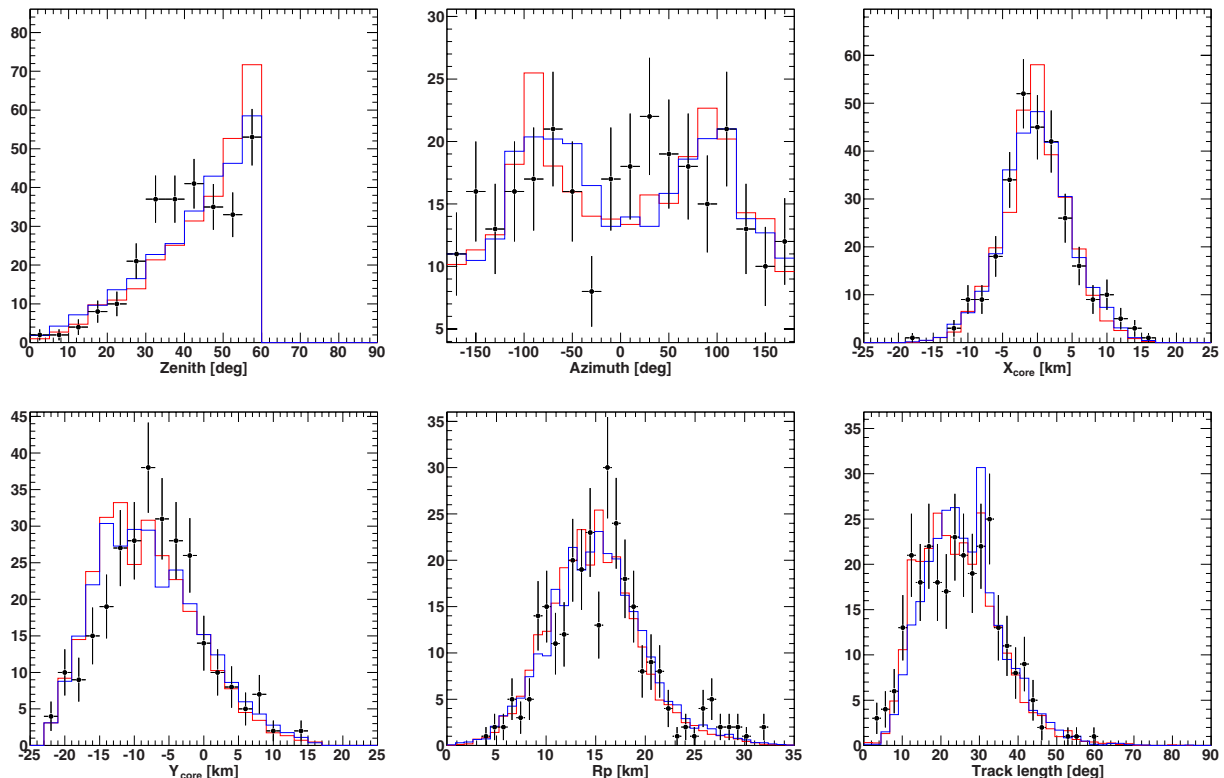

Figure 3. Comparison of the data and MC for TA FD stereo events: zenith angle (upper left), azimuth angle (upper middle), core location (upper right, lower left), impact parameter (lower middle) and track length (lower right).
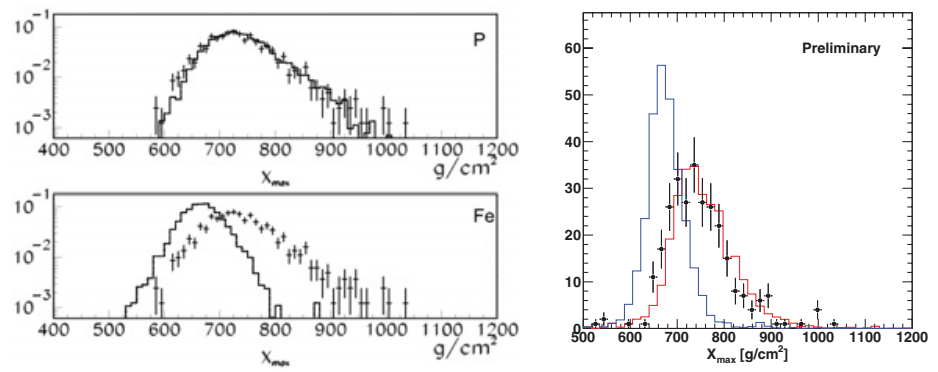

Figure 4. $X_{\max }$ distribution above the energy of $10^{18} \mathrm{eV}$ : HiRes (left), TA FD stereo (right). Points with error bar are data. Histograms are MC for proton (HiRes:upper, TA:red) and iron (HiRes:lower, TA:blue) primary.
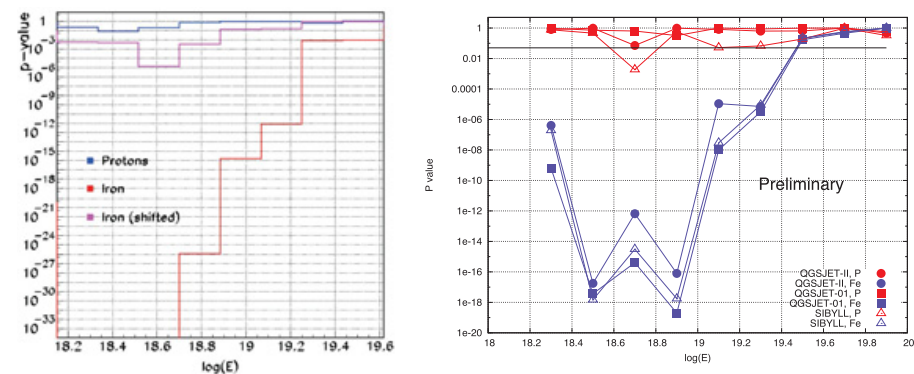

Figure 5. P-values estimated by KS test applied to $X_{\max }$ distribution for each primary particle type, hadronic interaction model and each energy region: HiRes (left), TA FD stereo (right). 

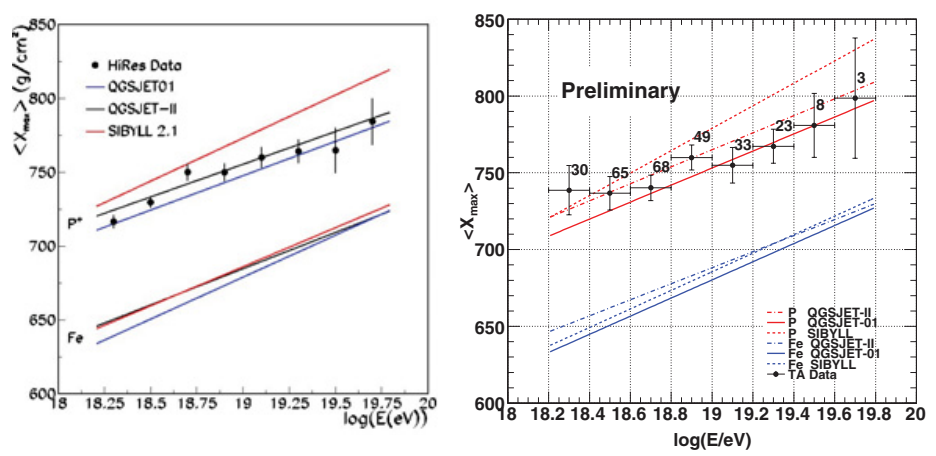

Figure 6. Averaged $X_{\max }$ of MC and Data. MC prediction rails of proton or iron primary include biases estimated by detector simulation: HiRes (left), TA FD stereo (right). Hadronic interaction models are QGSJET-01, QGSJET-II and SIBYLL.

The Telescope Array experiment is supported by the Japan Society for the Promotion of Science through Grantsin-Aid for Scientific Research on Specially Promoted Research (21000002) "Extreme Phenomena in the Universe Explored by Highest Energy Cosmic Rays", and the Inter-University Research Program of the Institute for Cosmic Ray Research; by the U.S. National Science Foundation awards PHY-0307098, PHY-0601915, PHY-0703893, PHY-0758342, and PHY-0848320 (Utah) and PHY-0649681 (Rutgers); by the National Research Foundation of Korea (2006-0050031, 2007-0056005, 2007-0093860, 2010-0011378, 2010-0028071, R32-10130); by the Russian Academy of Sciences, RFBR grants 10-02-01406a and 11-02-01528a (INR), IISN project No. 4.4509 .10 and Belgian Science Policy under IUAP VI/11 (ULB). The foundations of Dr. Ezekiel R. and Edna Wattis Dumke, Willard L. Eccles and the George S. and Dolores Dore Eccles all helped with generous donations. The State of Utah supported the project through its Economic Development Board, and the University of Utah through the Office of the Vice President for Research. The experimental site became available through the cooperation of the Utah School and Institutional Trust Lands Administration (SITLA), U.S. Bureau of Land Management and the U.S. Air Force. We also wish to thank the people and the officials of Millard County, Utah, for their steadfast and warm support. We gratefully acknowledge the contributions from the technical staffs of our home institutions and the University of Utah Center for High Performance Computing (CHPC).

\section{References}

[1] T. Fujii et al., AIP Conf. Proc. 1367 (2011) 149

[2] T. Tomida et al., NIM A $\mathbf{6 5 4}$ (2011) 653

[3] D. Heck et al., Report FZKA 6019, (1998)

[4] N.N. Kalmykov and S.S. Ostapchenko, Phys. At. Nucl. 56 (1993) 346

[5] S. Ostapchenko, Nucl. Phys. B, Proc. Suppl. 151 (2006) 143

[6] R.S. Fletcher, T.K. Gaisser, P. Lipari, and T. Stanev, Phys. Rev. D50 (1994) 5710

[7] R. Engel, T.K. Gaisser, P. Lipari, and T. Stanev, Phys. Rev D46 (1992) 5013 